\title{
A comparison between the Static Balance Test and the Berg Balance Scale: validity, reliability, and comparative resource use
}

Clinical Rehabilitation

2016, Vol. 30(3) 288-293

(C) The Author(s) 2015

Reprints and permissions:

sagepub.co.uk/journalsPermissions.nav

DOI: $10.1177 / 0269215515578297$

cre.sagepub.com

@SAGE

\author{
Heidrun Maria Pickenbrock', Andrea Diel ${ }^{2}$ \\ and Antonia Zapf ${ }^{3}$
}

\begin{abstract}
Objective: Within a sample of acute post-stroke patients, to compare the score on the Berg Balance Scale and the Static Balance Test for validity, inter-rater reliability, and the expenditure of time.

Design: Prospective, intra-individual, cross-sectional evaluation study.

Setting: Acute stroke unit of a university hospital in Germany.

Participants: A total of 53 patients with acute stroke who did not have other pathology affecting their balance.

Main outcome measure: For intra-individual comparisons of the Berg Balance Scale and the Static Balance Test, Pearson correlation coefficients were calculated. For inter-rater reliability, Bland Altman plots were drawn and the corresponding mean difference and limits of agreement were calculated.

Results: The Static Balance Test took three to five minutes; the Berg Balance Scale 20-30 minutes. There was a high correlation between the scores on the Berg Balance Scale and the Static Balance Test $(r=0.91)$. For the Berg Balance Scale, the mean difference between the two raters was 0.13 and the limits of agreement were small $(-0.25 ; 0.5 \mathrm{I})$. For the Static Balance Test, the mean difference between the two raters was -0.02 and also the limits of agreement $(-0.06 ; 0.02)$ were even smaller than for the Berg Balance Scale. Both scales showed excellent inter-rater reliability.

Conclusion: The Static Balance Test was compared with the Berg Balance Scale and turned out to be equally valid, more reliable, and takes much less time. For the moment, the scale can be recommended for the use in acute stroke care, especially for the daily routine therapy.
\end{abstract}

\section{Keywords}

Stroke, balance, assessment, Berg Balance Scale, rehabilitation

Received: 16 December 2014; accepted: 28 February 2015

'Hanover Medical School, Hannover, Germany

¿University Medical Center Mainz, Mainz, Germany

3University Medical Center Goettingen, Goettingen, Germany
Corresponding author:

Heidrun Maria Pickenbrock, Hanover Medical School, Carl-

Neuberg-Str. I, Hannover 30625, Germany.

Email: pickenbrock.heidrun@mh-hannover.de 


\section{Introduction}

Two to four weeks after a stroke, $83 \%$ of patients have difficulties with postural control and balance. ${ }^{1}$ The measurement of balance is important to help establish a prognosis, estimate the severity of the stroke, establish an exact treatment plan, and evaluate the success of the therapeutic outcome. ${ }^{2}$ The Berg Balance Scale is regarded as one of the gold standards for measuring balance. ${ }^{3}$ However, it is time-consuming (15-20 minutes) and has a floor and a ceiling effect. ${ }^{4,5}$

Scales are best for daily routine rehabilitation if they are easy, quick to run, and cheap. ${ }^{6}$ The recently developed Static Balance Test meets exactly these criteria. The test is hierarchically drafted using only vertical static positions - sitting as the easiest upright position, to tandem stance as the most difficult condition. Each position may be tested in two sensory conditions, eyes open and eyes closed, assuming the dependency on vision is a crucial criterion in testing balance. Testing only has to be executed in the estimated range of the ability of the patient (see Appendix). The purpose of this study was to investigate the psychometric properties of the Static Balance Test compared with the Berg Balance Scale.

\section{Methods}

\section{Subjects}

A sample of stroke subjects was recruited from an acute care facility, one to eight days after stroke. Adult patients were included if they had an acute first-time stroke with paresis. Exclusion criteria were secondary diseases that could affect balance, premorbid balance problems, confinement to bed, confusion, or aphasia that prevents informed consent. The test administrator reviewed the medical records for exclusion criteria and conducted the data collection. The modified Rankin Scale was used to illustrate the disability status. Patients or their custodians provided written informed consent prior to the study. The tests took place at the bedside of the patient to guarantee standardized conditions. The study was approved by the research ethics committee of Mayence (16 April 2013, case number 837.083 .13 [8768-F]).

\section{Procedure}

To establish a standardized procedure, two physiotherapists had been trained for three hours, including a trial run with six patients. According to published methods, the testing was administered on the same date. ${ }^{7}$ The sequence of Static Balance Test and Berg Balance Scale was randomly determined. One physiotherapist conducted the two tests while both raters assessed the observations on all patients. Simultaneous testing was undertaken in order to reduce the variability from repeating the tests on different occasions. To ensure blinding, there was no discussion between raters. The time needed was estimated after the whole testing.

\section{Statistical analysis}

A total of 53 patients were included in this study, a number similar to comparable studies. ${ }^{8}$ The baseline characteristics were displayed using descriptive measures. To investigate the correlation between the two balance tests and the baseline characteristics the Mann-Whitney $U$-test (2 groups) or the Kruskal-Wallis test ( $>2$ groups) was used. For the intra-individual comparison of the Berg Balance Scale and the Static Balance Test, scatter plots were drawn and Pearson correlation coefficients were calculated. For the inter-rater reliability, Bland Altman analyses were performed, including plots and calculation of the mean difference and limits of agreement. ${ }^{9}$

\section{Results}

From March to July 2013, 143 patients were admitted to the stroke unit and screened for eligibility. A total of 53 patients met the study characteristics and agreed to participate in the study. Of the patients, $28(53 \%)$ were female, 25 (47\%) male. Of the patients, $24(45 \%)$ had a lesion of the right hemisphere, $24(45 \%)$ a left-sided lesion, and five $(10 \%)$ patients experienced a lesion on both sides. The median time after stroke was three days (25\%/75\% quantiles: $2 / 4$ days). The mean (SD) age of the subjects was 70 (11) years (range 50 $86)$. In terms of disability, 27 (51\%) patients were 


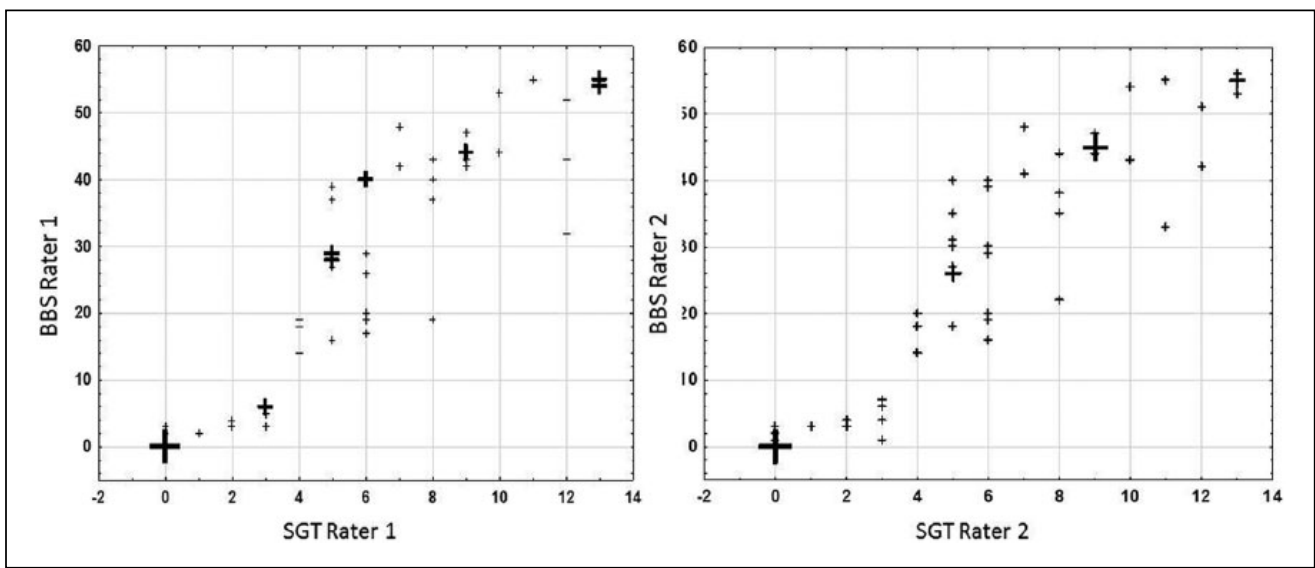

Figure I. Scatterplots for the comparison of the Berg Balance Scale and Static Balance Test for Rater I and 2 (the size of the markers corresponds to the frequency of the observed combination).

BBS: Berg Balance Scale; SGT: Static Balance Test.

severely affected (modified Rankin scale 5-4), 10 (19\%) moderately (modified Rankin scale 3 ), and 16 (30\%) mildly disabled (modified Rankin scale 2-1). The time to administer the Static Balance Test took 3-5 minutes; for the Berg Balance Scale 20-30 minutes. The Berg Balance Scale score as well as the Static Balance Test (both as the mean of the two raters) were not significantly different for males and females $(P=0.23, P=0.32)$, patients with lesions in the right or left hemisphere $(P=0.99$, $P=0.59)$, number of days post-stroke $(P=0.42$, $P=0.29)$, or between those $\leqslant 70$ or $>70$ years $(P=0.94, P=0.70)$. However, there was a clear correlation of both scores with the modified Rankin scale $(P<0.001$ both) with the more impaired patients with a lower score value.

Figure 1 illustrates a high correlation coefficient between the Static Balance Test and Berg Balance Scale for both raters, with the Pearson correlation coefficient 0.91 for Rater 1 as well as for Rater 2 .

Both tests showed high inter-rater reliability (see Figure 2). For the Berg Balance Scale, the mean difference was 0.13 , which corresponds to $0.2 \%$ of the scale range. The limits of agreement were smaller than \pm 1 point $(-0.25,0.51)$. For the Static Balance Test, the mean difference was equal to -0.02 , which corresponds to $0.1 \%$ of the scale range. The limits of agreement $(-0.06 ; 0.02)$ were even smaller than the limits of agreement for the Berg Balance Scale.

\section{Conclusion}

In this study, the psychometric properties of the Static Balance Test were compared with the Berg Balance Scale. Both tests had similar, excellent inter-rater reliability and concurrent validity. However, the Static Balance Test can be undertaken in four minutes, rather than 25 minutes on average.

The Berg Balance Scale is regarded as the gold standard, but in our study it took even more time (20-30 minutes) than reported in other studies. In contrast, the Static Balance Test only takes 3-5 minutes. This is because the test uses less items and it only has to be carried out in the ability range of the patient. Hence, using this assessment can increase the effectiveness of physiotherapeutic assessment and allows more time for treatment.

The two tests used in this study have different hypothetic approaches. The Static Balance Test is based on four static conditions (sitting and standing in different, difficult double foot support positions). If a position can be maintained for 30 seconds it will be tested with eyes closed. The Berg Balance Scale evaluates performance on 14 different tasks, thereby 

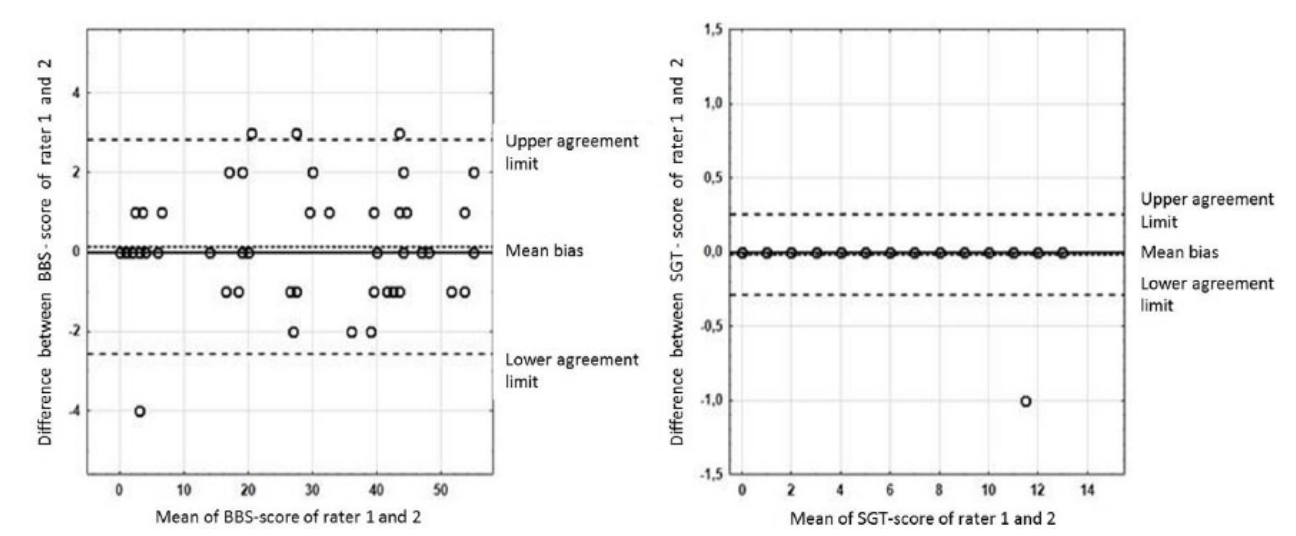

Figure 2. Bland Altman plots for the Berg Balance Scale and Static Balance Test (the range of the $y$-axis is in both cases $\pm 10 \%$ of the scale range).

BBS: Berg Balance Scale; SGT: Static Balance Test.

covering static (sitting, standing), dynamic (transfer) as well as challenging aspects of balance (picking something up from the floor). While the Berg Balance Scale is more comprehensive, it requires more time to administer.

Balance is a complex motor skill derived from the interaction of multiple sensorimotor processes. ${ }^{10}$ While standing, non-disabled adults tend to use somatosensory information up to $70 \%$ and vision about $10 \%$. Older adults show more use of visual information than younger adults. ${ }^{11}$ In poststroke patients, the use of somatosensory input can shift to excessive dependency on visual cues. ${ }^{12}$ Therefore, the Static Balance Test consequently tests each condition with closed eyes to eliminate this input. Our results indicate that there might be a measurable correlation between the performance of different tasks and these blindfolded conditions. Whether this is a true correlation has to be ascertained in another study, investigating whether the Static Balance Test can predict the risk of falling.

Some limitations of the study have to be considered. The patients were recruited only in one centre, a multicentre study would lead to greater confidence in the external validity. The study setting only allowed the referral of patients from an acute stroke unit. There was no follow-up of the subjects. Further, the sample of subjects included $51 \%$ with a modified Rankin Scale of 4-5, which is slightly higher than reported by Hankey (44\% severely disabled acute post-stroke). ${ }^{13}$ Therefore the results of this study are somewhat more valid for more strongly disabled subjects acute post-stroke.

The whole testing procedure in this study lasted around half an hour. During this time both learning and fatigue could not be excluded. Based on concerns of fatigue, we decided against immediate repetition of the tests, as different results could have occurred given spontaneous change in signs and symptoms, and subtle recovery can be expected from day to day in patients who are in the acute stage post-stroke. Thus, this study did not evaluate intra-rater reliability. The inter-rater reliability is only valid for the reproducibility of the scoring, as the same person always conducted the tests, in contrast to multiple testers in research or clinical practice. Multiple testers as a source of variability were not evaluated in this study.

Recently Suzuki et al. studied the correlation between another Static Balance Test and the Berg Balance Scale. Using different conditions (instead of blind folding lateral flexing of the trunk within different postures), they found a high correlation. ${ }^{14}$

The Static Balance Test had excellent qualities compared with the Berg Balance Scale in terms of validity and reliability. At the same time, the Static 
Balance Test has a significant advantage over the Berg Balance Scale: it is a very quick measurement. In future studies, it is worth assessing the Static Balance Test with respect to different disability scales, like the Barthel Index or Functional Independence Measure, particularly focusing on the floor and ceiling effects, the predictive power of the tests, as well the application of the test to chronic post-stroke patients or patients with other acute and chronic central nervous system lesions.

\section{Clinical messages}

- The Static Balance Test is comparable with the Berg Balance Scale in terms of validity and shows excellent reliability.

- The Static Balance Test can be undertaken in about four minutes, while the Berg Balance Scale takes 25 minutes on average.

\section{Conflict of interest}

The authors declare that there is no conflict of interest.

\section{Funding}

This research received no specific grant from any funding agency in the public, commercial, or not-for-profit sectors.

\section{References}

1. Tyson S, Hanley M and Chillala J. Balance disability after stroke. Phys Ther 2006; 86: 30-38.

2. Nichols DS, Miller L, Colby LA and Pease WS. Sitting balance: its relation to function in individuals with hemiparesis. Arch Phys Med Rehabil. 1996; 77: 865-869.
3. Tyson S and Connell L. How to measure balance in clinical practice. A systematic review of the psychometrics and clinical utility of measures of balance activity for neurological conditions. Clin Rehabil 2009; 23: 824-840.

4. Schädler S, et al. Assessments in der Rehabilitation. Band 1: Neurologie. 3rd ed. Bern: Hans Huber, 2012.

5. Blum L and Korner-Bitensky N. Usefulness of the Berg Balance Scale in stroke rehabilitation: A systematic review. Phys Ther 2008; 88: 559-566.

6. Hsueh IP, Chen JH, Wang $\mathrm{CH}$, et al. Development of a computerized adaptive test for assessing balance function in patients with stroke. Phys Ther 2010; 90: 1336-1344.

7. Verheyden G, Nieuwboer A, Mertin J, et al. The Trunk Impairment Scale: A new tool to measure motor impairment of the trunk after stroke. Clin Rehabil 2004; 18 : 326-334.

8. Schmid AA, Van Puymbroeck M, Altenburger PA, et al. Balance is associated with quality of life in chronic stroke. Top Stroke Rehabil 2013; 20: 340-346.

9. Bland JM and Altman DG. Statistical methods for assessing agreement between two methods of clinical measurement. Lancet 1986; 1: 307-310.

10. Horak FB. Postural orientation and equilibrium: What do we need to know about neural control of balance to prevent falls? Age and Ageing 2006; 35(Suppl 2): ii7-ii11.

11. Yeh TT, Cluff T and Balasubramaniam R. Visual reliance for balance control in older adults persists when visual information is disrupted by artificial feedback delays. PloS One 2014; 9: e91554.

12. Bonan IV, Colle FM, Guichard JP, et al. Reliance on visual information after stroke. Part I: balance on dynamic posturography. Arch Phys Med Rehabil 2004; 85: 268273.

13. Hankey GJ, Spiesser J, Hakimi Z, et al. Rate, degree, and predictors of recovery from disability following ischemic stroke. Neurology 2007; 68: 1583-1587.

14. Suzuki M, Fujisawa H, Machida Y, et al. Relationship between the Berg Balance Scale and Static Balance Test in hemiplegic patients with stroke. J Phys Ther Sci 2013; 25: 1043-1049. 


\section{Appendix}

\section{Static Balance Test}

Testing is conducted only in the range of the patient's functional level to his/her maximum (e.g. severely disabled: from 0 to 5 , mildly affected from about 9 ).

If a patient does not pass a level with eyes closed he/she will not be tested in the next level. A condition is tested only once.

The tester stands beside the patient to give support if necessary.

0-3: Thighs completely supported, not necessarily the feet. Patient is sitting independently not using the environment to hold on.

4-15: Patient is standing sideward to a bed or a therapy table, his/her less involved side toward the bed. For foot placement see schemes below.

10-15: The more involved foot is backward; the center of mass is above this foot.

0 Not able to sit independently at the edge of the bed

I Independent sitting at the edge of the bed

2 Independent sitting at the edge of the bed

3 Independent sitting at the edge of the bed

4 Independent standing, feet spaced out at the width of the pelvis

5 Independent standing, feet spaced out at the width of the pelvis

6 Independent standing, feet spaced out at the width of the pelvis

7 Independent standing, feet closed

8 Independent standing, feet closed

9 Independent standing, feet closed

10 Independent standing, modified tandem stand*

II Independent standing, modified tandem stand*

12 Independent standing, modified tandem stand*

13 Independent standing, tandem stand**

14 Independent standing, tandem stand ** $^{*}$

15 Independent standing, tandem stand ${ }^{* *}$ eyes open at least 30 seconds

eyes closed less than 30 seconds

eyes closed more than 30 seconds

eyes open

eyes closed

eyes closed

eyes open

Eyes closed

eyes closed

eyes open

eyes closed

eyes closed

eyes open

eyes closed

eyes closed at least 30 seconds

less than 30 seconds

more than 30 seconds

at least 30 seconds

less than 30 seconds

more than 30 seconds

at least 30 seconds

less than 30 seconds

more than 30 seconds

at least 30 seconds

less than 30 seconds

more than 30 seconds

* standing with one foot ahead the other, feet apart as if closed.

** standing with one foot aligned ahead the other. 